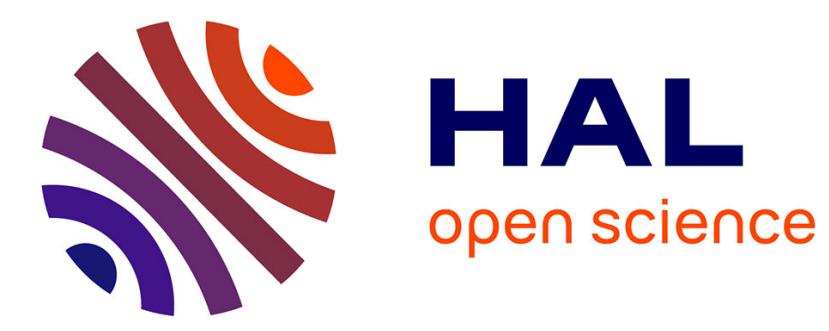

\title{
Two numerical investigations of the three-state chiral Potts model
}

\author{
Jean-Christian Anglès d'Auriac, Jie Chen, J. Maillard
}

\section{To cite this version:}

Jean-Christian Anglès d'Auriac, Jie Chen, J. Maillard. Two numerical investigations of the threestate chiral Potts model. Journal de Physique I, 1993, 3 (2), pp.567-578. 10.1051/jp1:1993150 . jpa-00246741

\section{HAL Id: jpa-00246741 https://hal.science/jpa-00246741}

Submitted on 1 Jan 1993

HAL is a multi-disciplinary open access archive for the deposit and dissemination of scientific research documents, whether they are published or not. The documents may come from teaching and research institutions in France or abroad, or from public or private research centers.
L'archive ouverte pluridisciplinaire HAL, est destinée au dépôt et à la diffusion de documents scientifiques de niveau recherche, publiés ou non, émanant des établissements d'enseignement et de recherche français ou étrangers, des laboratoires publics ou privés. 
Classification

Physics Abstracts

$05.50-05.70$

\title{
Two numerical investigations of the three-state chiral Potts model
}

\author{
J.C. Anglès d'Auriac(*), J. Chen(*) and J.M. Maillard(**) \\ (') Centre des Recherches sur les Très Basses Températures, BP 166, 38042 Grenoble, France \\ $\left({ }^{* *}\right)$ Laboratoire de Physique Théorique et Hautes Energies, Boite 126 Tour 16,1er étage, 4 place \\ Jussieu, 75252 Paris Cedex 05, France
}

(Received 12 September 1992, accepted 30 September 1992)

\begin{abstract}
Résumé. - Le diagramme de phase du modèle de Potts chiral à trois états est étudié numériquement par deux méthodes différentes. Une attention particulière est portée par simulation Monte Carlo au point d'intersection entre la ligne self-duale et la courbe d'intégrabilité du modèle. En outre le mécanisme donnant lieu à la multiplicité des maxima de la chaleur spécifique comme fonction de la température est expliqué, au moins pour les petites tailles, par des considérations sur la structure de la densité d'états. L'interprétation physique des courbes d'intégrabilité reste une question ouverte.
\end{abstract}

\begin{abstract}
The phase diagram of the three-state chiral Potts model on a square lattice is numerically investigated using two different methods. The special point located at the intersection of the self-dual line and the integrability curve is analyzed in great detail using extensive Monte-Carlo simulations. The mechanism giving rise to the multiplicity of the maxima of the specific heat as a function of the temperature, is explained, at least for small sizes, by considering the structure of the density of states. The physical interpretation of the integrability curves still remains an open question.
\end{abstract}

\section{Introduction.}

The chiral Potts model is an interesting "toy model" which helps one in understanding important physical issues such as commensurate-incommensurate transitions, floating phases, or the occurrence of very rich phase diagrams even for two-dimensional systems [1]. The physics emerging from chirality is still far from being understood. Moreover, a better understanding of the level crossing problems in statistical mechanics seems to be a necessary step for many modern solid state physics problems (High $T_{\mathrm{c}},{ }^{\circ}$ ). Recently interest in this model was rekindled by the discovery of new solutions of the Yang-Baxter equations for this model [2, 3]. These integrability cases happened to be the first solutions of the Yang-Baxter equations with genus greater than one [4]. In the last year particular efforts have been devoted specifically to 
the three-state chiral Potts model [5]. Despite its smaller number of parameters, this model exhibits a rich phase diagram and many subtleties [6].

We first briefly recall the integrability curves, the self-dual lines and the symmetries of this model on a square lattice. We then introduce trajectories with fixed chirality. These particular trajectories in the phase diagram enable a better understanding of the physics of the model. We take two approaches in this letter. First, exact partition functions for small lattice sizes are calculated by exhaustive enumeration. Then, extensive Monte-Carlo simulations are performed for much larger sizes, with a special emphasis on two-point correlation functions. It is known that size effects are very strong for this model [7]. It is also very sensitive to boundary conditions. Rather than being a problem, this sensitivity happens here to be an advantage: using different boundary conditions, we identify on small lattices different properties of the thermodynamical limit. Its is striking to find on small lattice sizes, such as the $4 \times 4$ lattice, two maxima for the specific heat as a function of the temperature. The peculiar form of the density of states well explains the occurrence of these two maxima. On the other hand, quantities such as correlation functions require large sizes and can only be investigated by Monte Carlo simulations. We find an oscillatory behaviour for the two spin correlation function as a function of the distance between the two spins. This can be seen as correlated with the occurrence of a floating phase for the model.

\section{The model.}

The partition function of the isotropic three-state chiral Potts model on a square lattice reads:

$$
Z=\sum_{\sigma} \prod_{\langle i, j\rangle} w\left(\sigma_{i}-\sigma_{j}\right)
$$

where $\sigma_{i}=0,1,2$, the difference is taken modulo 3 , the product runs over all the vertical and horizontal bonds and the sum runs over all the spin configurations. The parameter space is spanned by the three homogeneous parameter $w(0), w(1)=w(-2)$ and $w(2)=w(-1)$. This three-parameter homogeneous space will be described in terms of two independent variables. We will use the $(a-b)$ parameter space where

$$
\begin{gathered}
a=(w(0)-2 w(1)+w(2)) /(\sqrt{6}(w(0)+w(1)+w(2))) \\
b=(w(0)-w(2)) /(\sqrt{2}(w(0)+w(1)+w(2)))
\end{gathered}
$$

We will also make the chirality $\Delta$ explicit using a $(\Delta-T)$ plane, where

$$
\begin{gathered}
w(0)=\exp (\cos (2 \pi \Delta / 3) / T) \\
w(1)=\exp (\cos (2 \pi(\Delta+2) / 3) / T) \\
w(2)=\exp (\cos (2 \pi(\Delta+1) / 3) / T)
\end{gathered}
$$

This model is known in the literature as the CC3 model [9]. The spin reversal symmetry, $S: \sigma_{i} \rightarrow-\sigma_{i}$, yields an invariance of the partition function under the exchange of $w(1)$ and $w(2)$. In terms of the $(a-b)$ variables this accounts for a symmetry reflection with respect to the line $a=\sqrt{3} b$. This also amounts to negating the chirality $\Delta \rightarrow-\Delta$. Note that this invariance is compatible with any boundary conditions. Another symmetry, denoted $\mathrm{C}$, corresponding to a simple transformation of the dummy variables $\sigma_{i}$ also exists for lattice sizes which are multiples of three with periodic boundary conditions, and for lattices of any size with open boundary conditions. In terms of the homogeneous variables $w(i)$ 's the symmetry $\mathrm{C}$ reads 
$w(i) \rightarrow w(i+1)$. This transformation is of order three. The corresponding transformation in the $(a-b)$ plane is a $2 \pi / 3$ rotation, or a shift of the chirality $\Delta \rightarrow \Delta+1$ in the $(\Delta-T)$ plane. A "duality" symmetry D also exists for this model [6],[11]

$$
\mathrm{D}: \quad w(n) \rightarrow \widehat{w}(n)=\sum_{p} \omega^{n p} w(p)
$$

where $\omega^{3}=1$.

In fact it is a transformation of order four [8]. There exists a line globally invariant under the transformation $D$. This self-dual line reads :

$$
\sqrt{3} w(0)=w(0)+w(1)+w(2)
$$

Some integrability conditions of Au-Yang et al. for this model [8] correspond to the two branches in parameter space:

$$
\begin{array}{r}
\sqrt{3} P+Q=0 \\
Q=0
\end{array}
$$

where

$$
\begin{gathered}
P=f-3 h \\
Q=f-2 g+3 h \\
f=w(0) w(1) w(2) \cdot\left(w(0)^{3}+w(1)^{3}+w(2)^{3}\right) \\
g=w(0)^{3} w(1)^{3}+w(1)^{3} w(2)^{3}+w(0)^{3} w(2)^{3} \\
h=w(0)^{2} w(1)^{2} w(2)^{2}
\end{gathered}
$$

Others integrability conditions also exits specifically for the $q=3$ chiral Potts model [?] and are not represented in figure 1 . The triangle $\mathrm{ABC}$ of figure 1a represents in the $(a-b)$ plane the physical region where all the Boltzmann weights $w(0), w(1)$ and $w(2)$ are positive. The self-dual line and its transforms under the symmetry $\mathrm{C}$ are represented by full lines. The first branch $\sqrt{3} P+Q=0$ of the integrability curve is the dashed line joining $\mathrm{A}$ with $\mathrm{B}, \mathrm{B}$ with $\mathrm{C}$ and $\mathrm{C}$ with $\mathrm{A}$. The three dashed loops attached to $\mathrm{A}, \mathrm{B}$ and $\mathrm{C}$ respectively are the physical part of the second branch $Q=0$. The ferromagnetic standard scalar Potts model [13] corresponds to the AO line. The same lines are also shown with the same symbol in the $(\Delta-T)$ plane in figure $1 \mathrm{~b}$. The six regions of the phase diagram which map into each other by the symmetry groups generated by the transformation $\mathrm{C}$ and the transformation $\mathrm{S}$ are labelled by the same number in the $(a-b)$ plane and in the $(\Delta-T)$ plane. Figure 1c represents fixed chirality trajectories in the $(a-b)$ plane: namely $\Delta=0$ (the standard scalar Potts line), $\Delta=-0.2$, $\Delta=-0.3$ and $\Delta=-0.4$. The value $\Delta=-0.3$ was choosen because the corresponding fixed chirality trajectory almost contains the intersection $M$ of the self-dual line with the integrability curve $\sqrt{3} P+Q=0$. Recalling a previous Monte Carlo study of the three-state chiral Potts model [10], this very special point $M$ could be the bifurcation point where the floating phase originates, similarly to what happens for the Ashkin-Teller model [14] where the self-dual line splits into two critical curves.

\section{Enumeration results for small lattice size.}

Denoting $n_{\alpha}$ the number of bonds $(i, j)$ with $\sigma_{j}-\sigma_{i}=\alpha, \alpha=0,1,2$, we have calculated the total number $g\left(n_{0}, n_{1}, n_{2}\right)$ of spin configurations for each $n_{0}, n_{1}, n_{2}$. The partition function thus reads: 


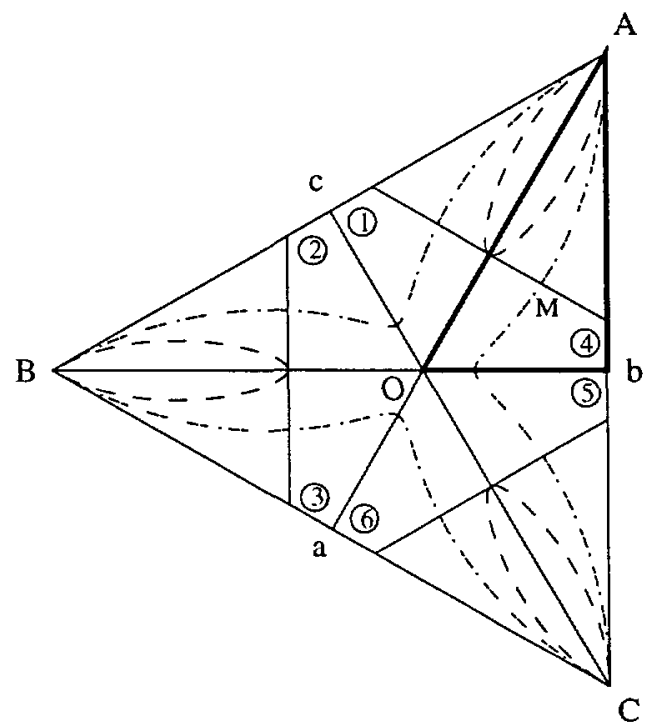

(a)

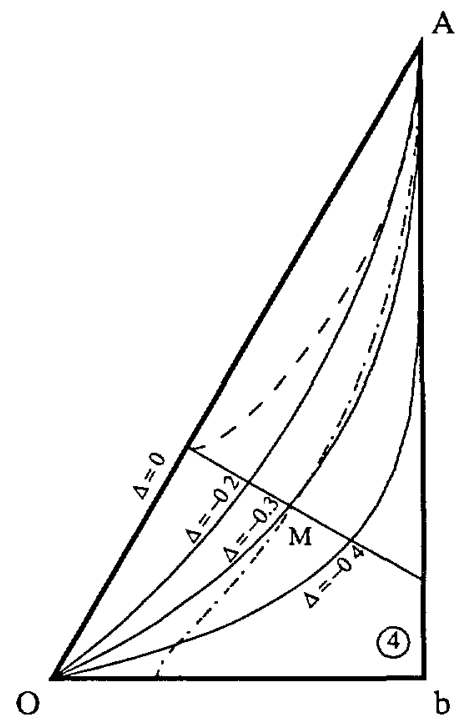

(b)

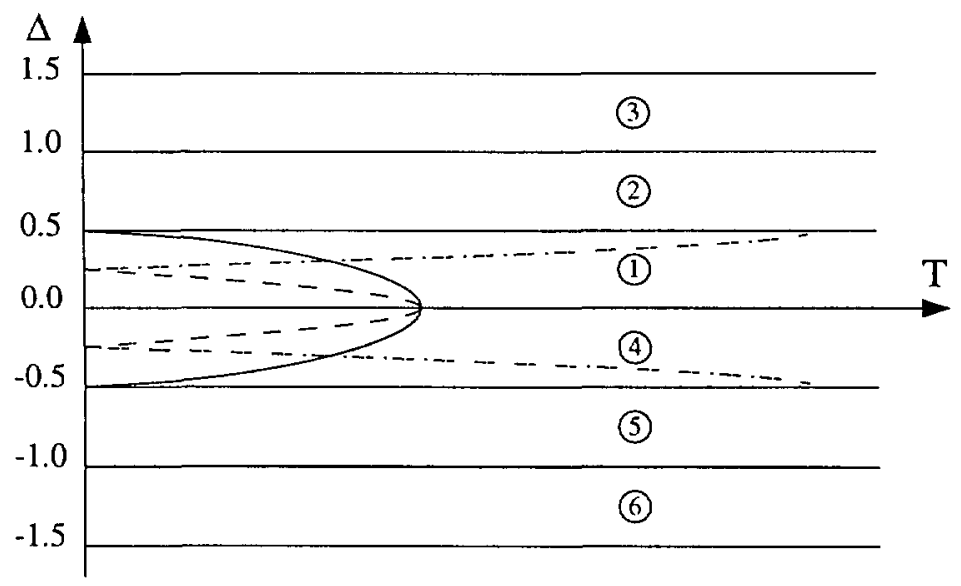

(c)

Fig.1. - Phase diagram of the three-state chiral Potts model on a square lattice (see text).

$$
Z=\sum_{n_{0}, n_{1}, n_{2}} g\left(n_{0}, n_{1}, n_{2}\right) \cdot w(0)^{n_{0}} w(1)^{n_{1}} w(2)^{n_{2}}
$$

It is straightfoward to calculate from all the $g\left(n_{0}, n_{1}, n_{2}\right)$ the partition function, the internal energy, the specific heat etc. for any point of the $(a-b)$ plane or of the $(\Delta-T)$ plane. The calculation was performed in four cases: a $4 \times 4$ square lattice with open boundary conditions, a $4 \times 4$ square lattice with periodic boundary conditions, a $6 \times 3$ rectangular lattice with open boundary conditions and a $6 \times 3$ rectangular lattice with periodic boundary conditions. Figure 


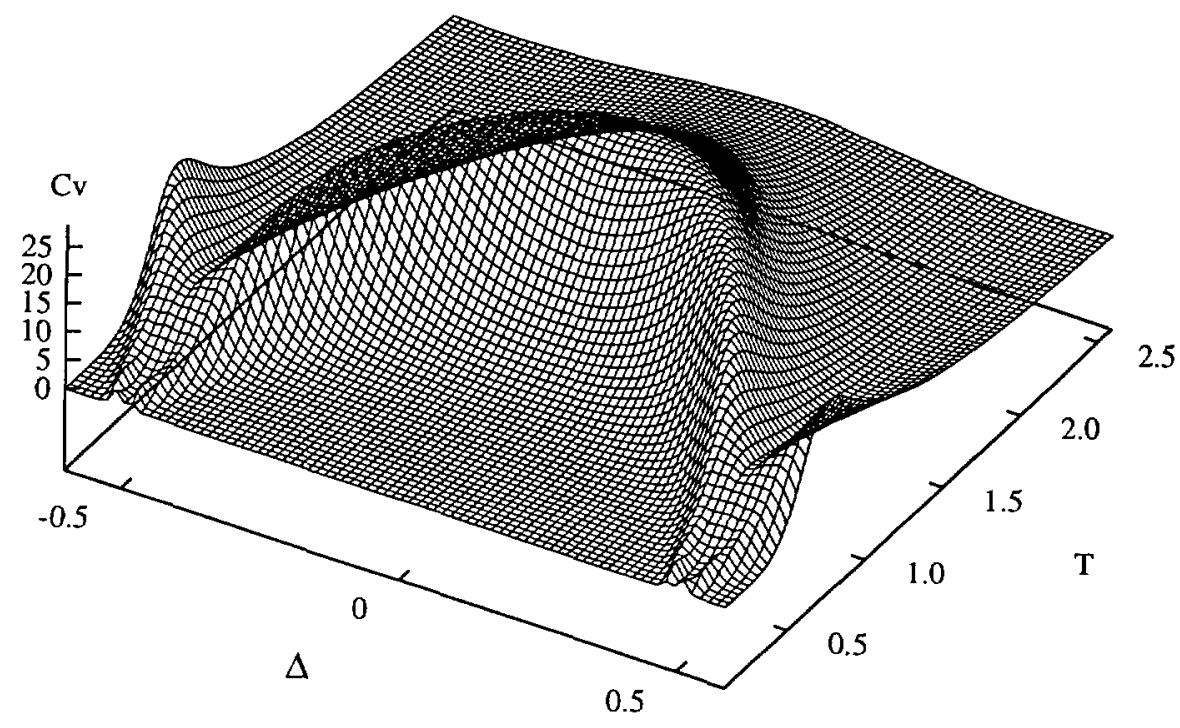

Fig.2. - Specific heat as a function of the chirality $\Delta$ and the temperature $T$ on a $6 \times 3$ rectangular lattice.

2 shows the specific heat as a function of the temperature $T$ and of the chirality $\Delta$ for a rectangular $6 \times 3$ lattice and periodic boundary conditions. For the other choices of lattice we obtained similar surfaces representing the specific heat as a function of $\Delta$ and $T$. For fixed chirality the existence of two maxima in the specific heat versus temperature is clearly seen for values of the chirality $\Delta$ ranging from 0.45 to 0.55 . This phenomena also occurs for the $4 \times 4$ periodic square lattice and is illustrated in figure $3 \mathrm{a}$. In this figure the bold line represents the specific heat with its two maxima. In order to understand the physical origin of these two maxima we analyzed the density of states. In figure $3 b$ the thin line shows for $\Delta=-0.47$ the logarithm of the density of states as a function of the energy. In the same figure the bold line represents the same density of states for $\Delta=-1 / 2$ where only 25 values of the Boltzmann factor are possible for any of the 43046721 states. For generic values of $\Delta$ the degeneracy comes from the fact that many configurations have the same triplet $\left(n_{0}, n_{1}, n_{2}\right)$ (the usual combinatorial degeneracy). Nevertheless for $\Delta=-1 / 2$ one also has a degeneracy of another origin: different triplets $\left(n_{0}, n_{1}, n_{2}\right)$ may give the same Boltzmann factor $w(0)^{n_{0}} w(1)^{n_{1}} w(2)^{n_{2}}$. Changing slighlty $\Delta$ from this $\Delta=-1 / 2$ value will lift this second type of degeneracy, and give its structure to the density of states for $\Delta=-0.47=-1 / 2+0.03$ as seen in figure $3 \mathrm{~b}$. The physical interpretation of the two maxima of figure $3 \mathrm{a}$ is now clear: for $T \geq 0.5$ the details of the density of states are irrelevant and the system orders the same way as for $\Delta=-1 / 2$, but for $T \leq 0.5$ the details of the density of states become important and the system orders among the numerous states which constitute the ground state for the $\Delta=-1 / 2$ case. This new ordering gives rise to the low-temperature maximum. This is illustrated in figure 3 where the dashed curve represents the specific heat for $\Delta=-1 / 2$. The broken line (low-temperature maximum) is obtained for $\Delta=-0.47$, taking into account only the fifteen lowest energy states. These fifteen states are located on the first band of the density of states for $\Delta=-0.47$ and have the same minimal energy for $\Delta=-1 / 2$ (see Fig. 3b). It is clearly seen in figure 3a that the bold curve is the sum of the two dashed and broken curves. Other values than $\Delta=-1 / 2$ may exhibits this property of "additional degeneracy". Figure 4 represents the ratio of triplets 


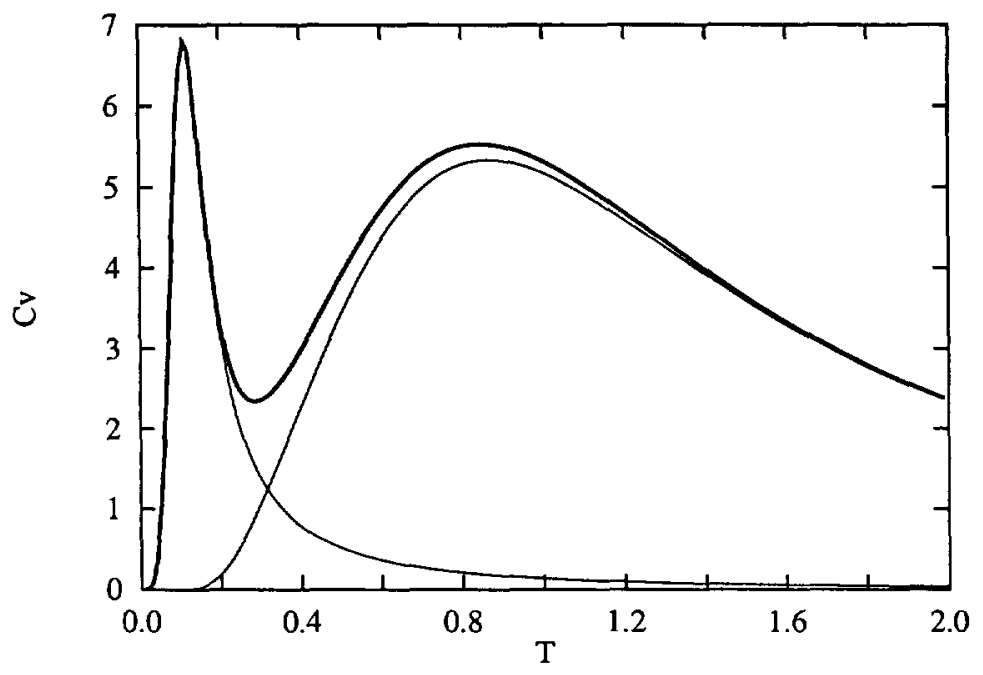

(a)

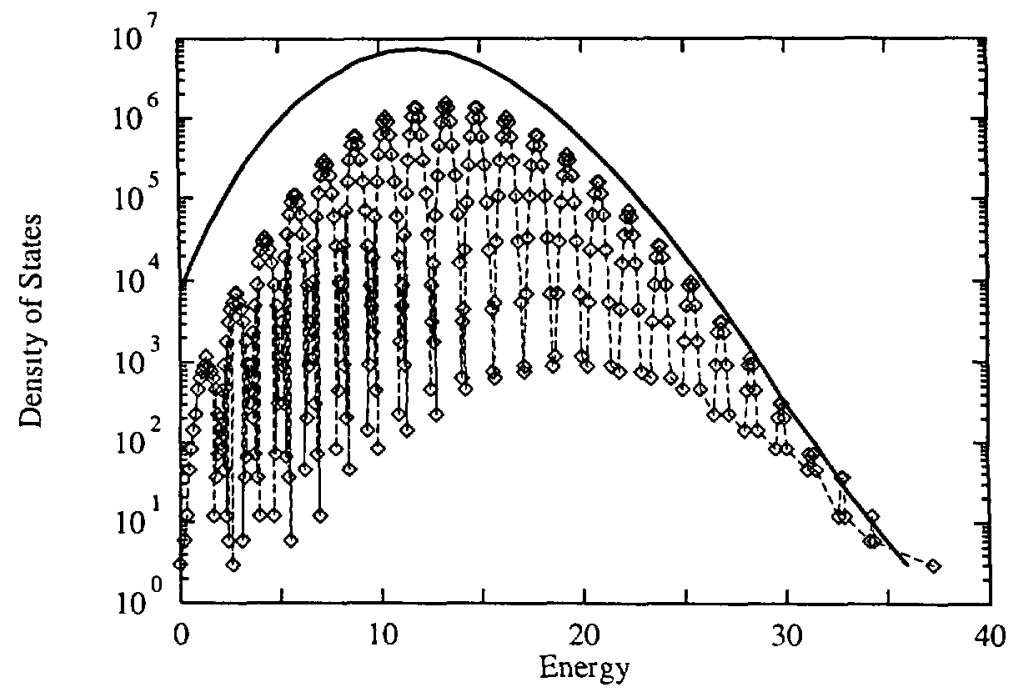

(b)

Fig.3. - a) Specific heat versus temperature for a $4 \times 4$ non periodic square lattice and $\Delta=-0.46$. b) Density of states for the same parameters (see text).

$\left(n_{0}, n_{1}, n_{2}\right)$ (with $n_{0}+n_{1}+n_{2}$ fixed) giving the same Boltzmann weight $w(0)^{n_{0}} w(1)^{n_{1}} w(2)^{n_{2}}$ to the total number of triplets as a function of $\Delta$. Among the values of $\Delta$ exhibiting this additional degeneracy, quarter integer values stand out. Indeed on a periodic $4 \times 4$ square lattice and for $\Delta=-7 / 4+\epsilon$ ( $\epsilon$ small) the specific heat versus temperature again exhibits two maxima. The location of the maxima of the specific heat in the $(\Delta-T)$ plane is given in figure 5 for a periodic $4 \times 4$ square lattice and for a periodic $6 \times 3$ rectangular lattice. Note that only the $6 \times 3$ lattice is invariant under the shift $\Delta \rightarrow \Delta+1 / 2$, due to the symmetry $C$ as already 


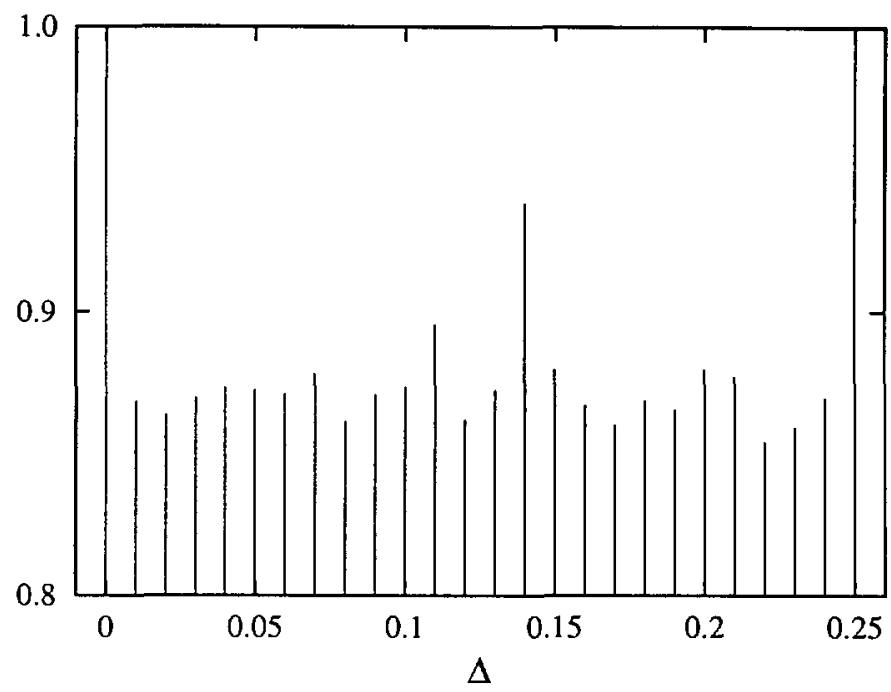

Fig.4. - Additionnal degeneracy for $n=n_{0}+n_{1}+n_{2}=2000$ versus $\Delta$ (see text).

explained. Also note that for this particular value of $\Delta$ the chiral Potts model has a residual entropy.

\section{Results of Monte Carlo simulations.}

Results of exact enumerations, though restricted to small sizes, enable one to find some features relevant even in the thermodynamical limit. However they do not give any hint about other quantities such as correlation functions. Let us therefore turn to Monte Carlo simulations with larger sizes [6] in order to revisit the phase diagram of the model and in particular to investigate the the extent of the floating phase. All our Monte Carlo simulations were performed on a square lattice with helicoidal boundary conditions in one direction and open boundary conditions in the other direction. We were able to reach equilibrium on a $128 \times 128$ lattice. We worked at fixed chirality which allowed us to have a criterion of equilibrium using the fluctuation-dissipation theorem.

One remaining question about the location of the floating phase of the three-state chiral Potts model is whether it originates at the (ferromagnetic) critical point $F$ of the standard scalar Potts model, or if one has a critical line (separating an ordered phase from a disordered phase) which would, at a certain point $Y$, split into two branches delimiting the floating phase (see Fig. 1). Previous Monte Carlo simulations showed that, up to the precision of this method, such a critical line, if it exists, would be indistinguishable from the self-dual line [6]. In this framework it is worthy to decide if the point $M$ ( intersection of the self-dual line with the integrability branches $\sqrt{3} P+Q=0)$ is not the bifurcation point $Y$. In the $(\Delta-T)$ plane point $\mathrm{M}$ has coordinates $\Delta=-0.304405$ and $T=1.08141$. We therefore chose the value $\Delta=-0.3$ to answer this question. We also chose $\Delta=-0.2$ as a comparison because this trajectory lies between point $M$ and point $F$. It is clear from our results that $M$ is not the bifurcation point $Y$. Indeed a floating phase exists over a finite range of temperature around $T=1.01841$. By contrast, for $\Delta=-0.2$, our accuracy is not good enough to decide wether or not the floating phase still exists. Figure 6 shows for $L=128$ and $\Delta=-0.3$ the specific heat as a function 


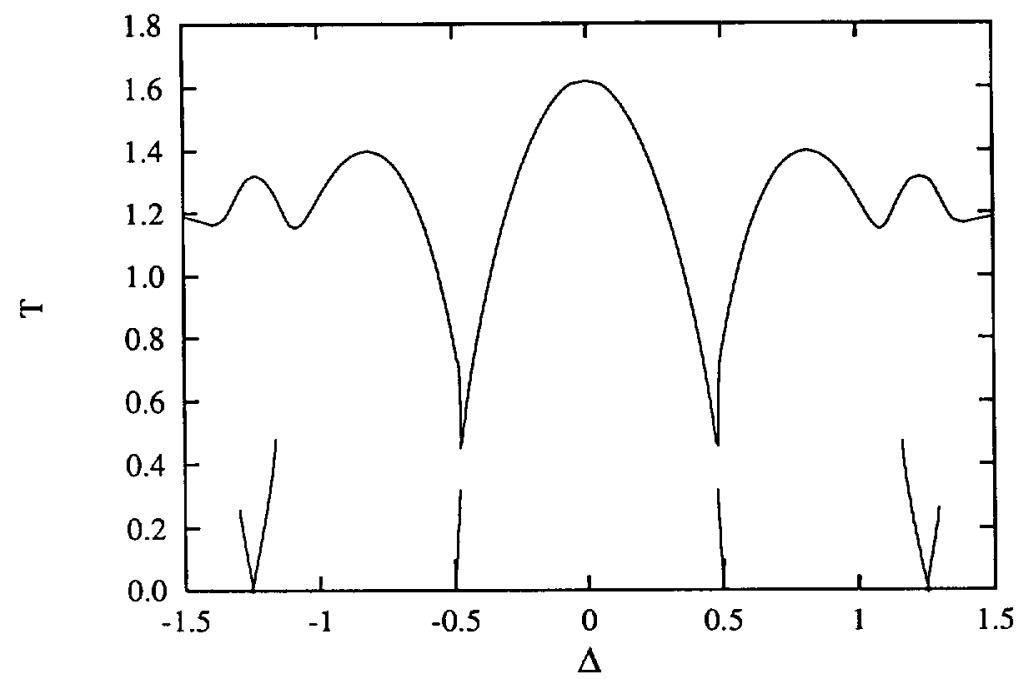

(a)

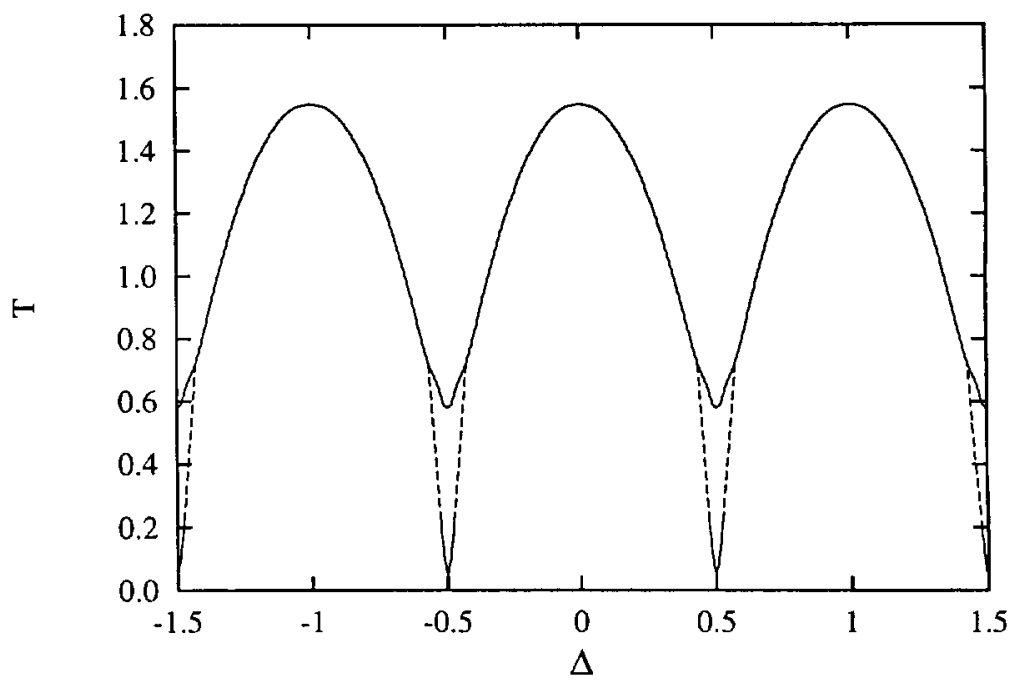

(b)

Fig.5. - Location of the maxima of specific heat in the $\Delta-T$ plane. a) for a $6 \times 3$ periodic rectangular lattice b) for a $4 \times 4$ periodic square lattice.

of the temperature. We easily identify five maxima (these maxima are reminiscent of the floating phase already encountered in previous simulations [6]). To understand the occurence of these maxima it is illuminating to look at snapshots of the sample for different temperatures below and above each maximum. For example we present in figure $7 \mathrm{a}$ and figure $7 \mathrm{~b}$ snapshots of $128 \times 128$ samples at $\Delta=-0.3$ for $T=0.95$ and $T=0.97$. The strips running diagonally accross the sample are evident. Due to the helicoidal boundary conditions, their number is a multiple of three. Passing through each maximum of the specific heat corresponds to an increment 


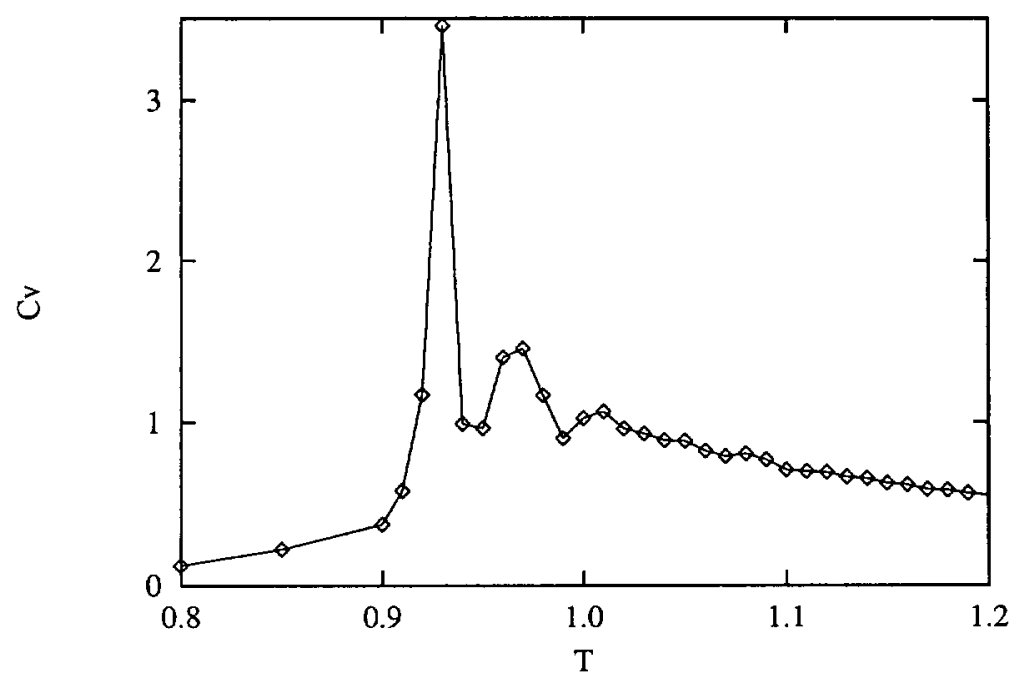

Fig.6. - Results of Monte Carlo simulation for the specific heat versus the temperature for a $128 \times 128$ square lattice with helicoidal boundary conditions and $\Delta=-0.3$.

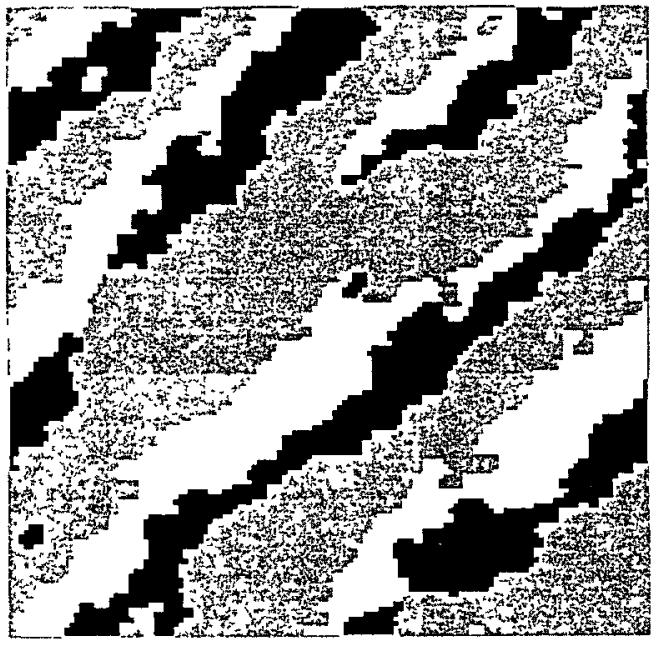

(a)

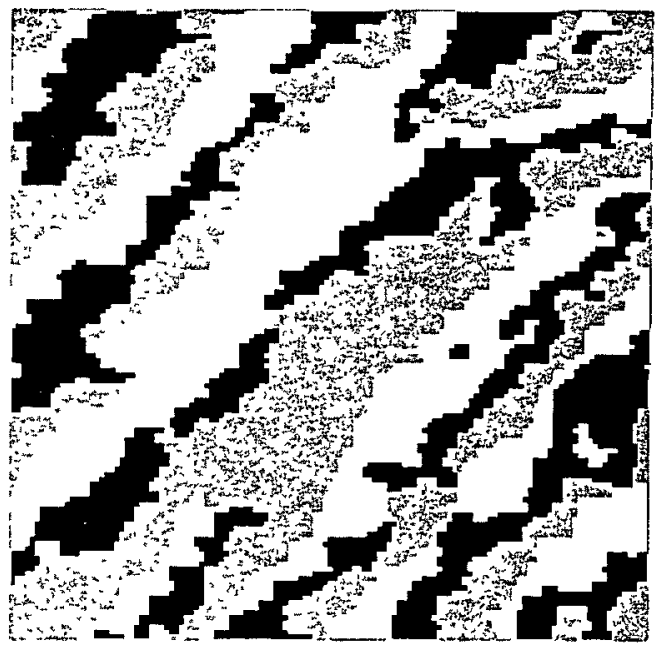

(b)

Fig.7. - Snapshots of the spins configuration for a $128 \times 128$ square lattice with helicoidal boundary conditions for $\Delta=-0.3$ and for: a) $T=0.95$ b) $T=0.97$.

by three of the number of strips. A more quantitative description of these strips can only be achieved by means of averaged quantities. In figure 8 we show, for $\Delta=-0.3$ and $T=0.97$, the most probable of the three values 0,1 or 2 for the spin $\sigma_{i}=\sigma_{(\alpha, \alpha)}$ given $\sigma_{(0,0)}=0$, as a function of the distance $\alpha$. We therefore restrict ourself to the diagonal perpendicular to the strip. There is no ambiguity on the number of strips on this averaged quantity. Finally different correlation functions can be defined. We defined for instance $P_{0}(\alpha)$ to be the probability that $\sigma_{(\alpha, \alpha)}-\sigma_{(0,0)}=0$ and $P_{1}(\alpha)$ to be the probability that $\sigma_{(\alpha, \alpha)}-\sigma_{(0,0)}=1$. The various correlation functions $\left\langle\sigma_{\alpha} \sigma_{0}\right\rangle$ which can be introduced can all be expressed in terms of $P_{0}(\alpha)$ 


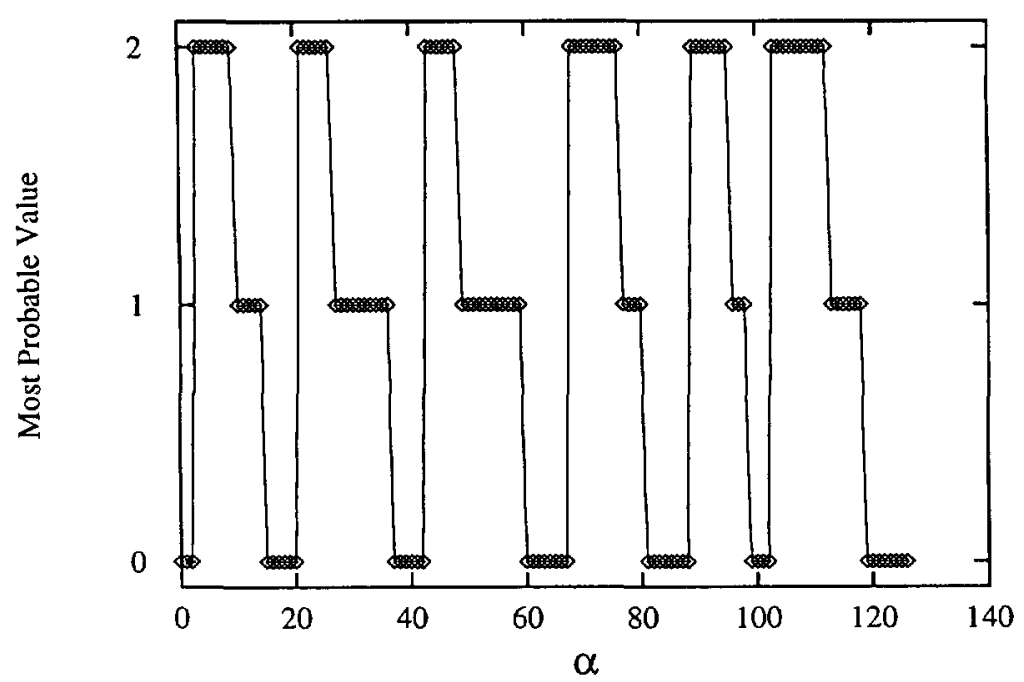

Fig.8. - Most probable value of the spin $\sigma_{\alpha}$ versus the distance $\alpha$ for a $128 \times 128$ square lattice (see text).

and $P_{1}(\alpha)$. Figure 9 a shows $P_{0}(\alpha)$ and $P_{1}(\alpha)$ for $\Delta=-0.30, L=64$ and $T=0.95$, as a function of the distance of $\alpha, 0 \leq \alpha \leq 128$, and figure $9 \mathrm{~b}$ shows the same quantities for $T=1.01$. Again the oscillations of the correlation functions are evident. Note that the two-points correlation functions along the diagonal parallel to the strip do not present oscillations.

\section{Conclusion.}

We have related the shape of the specific heat versus temperature curves to the density of states for the three-state chiral Potts model on a $4 \times 4$ square lattice. For integer, half integer and quarter integer values of the chirality an extra degeneracy of the ground state occurs. The low-temperature maximum of the specific heat for chirality close to these special values, is a consequence of the lifting of this extra degeneracy, while the high-temperature maximum comes from the "envelope" of the density of states. In others words one maximum is related to the general shape of the density of states while the other is related to the details of the first band of states. For larger sizes where the number of maxima increases, it would be interesting to see if this mechanism still holds. This could mean some kind of "hierarchical" structure for the density of states. In this point of view, the spectrum has a low energy band, and this band itself has a low energy sub-band etc..

On the other hand the characteristic oscillations of some correlation functions are in good agreement with the existence of a floating phase. Analysis of these correlation functions, and other quantities, gives much insight into the limit of this floating phase. Actually the particular point $M$, the intersection of the self-dual line with the integrability curve, was not found to identify with the bifurcation point where the floating phase originates. As a consequence the integrability branch $\sqrt{3} P+Q=0$ seems to cross the three regions of the phase diagram, in contrast with the branch $Q=0$ entirely located inside the ordered phase. What can be the physical interpretation of such "special" points occuring in the disordered phase (such as part of the the branch $\sqrt{3} P+Q=0$ in the disordered phase) One possibility is that 


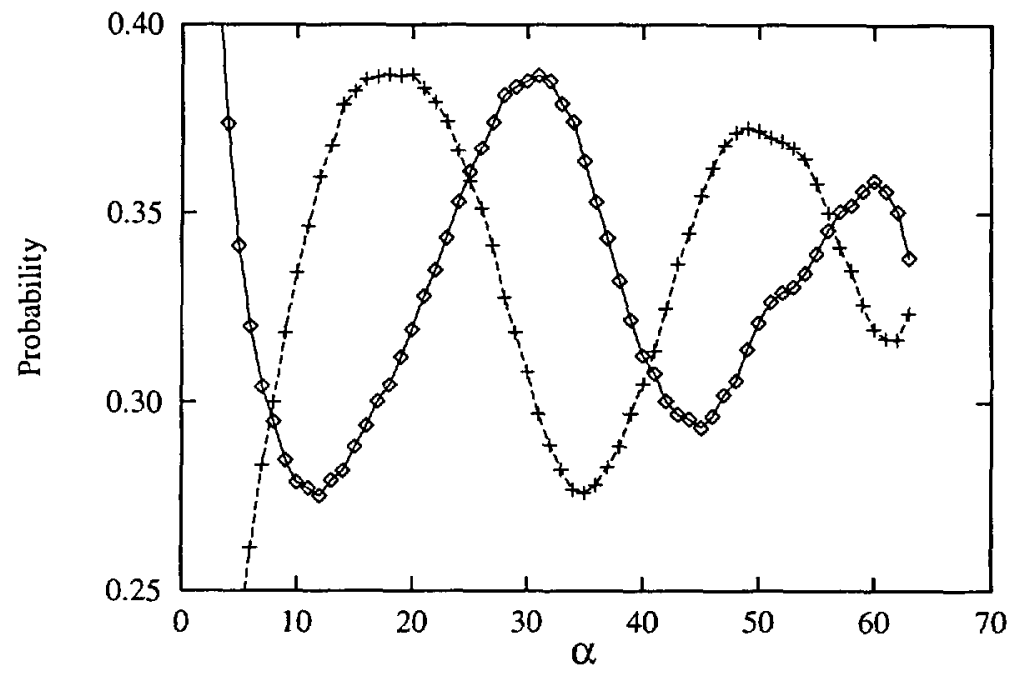

(a)

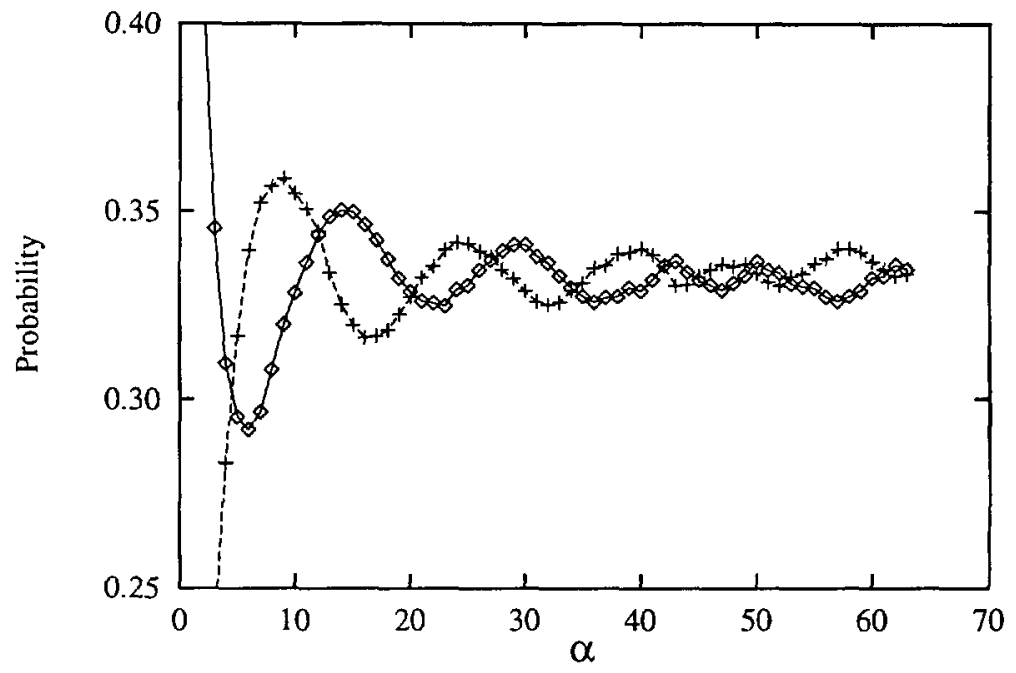

(b)

Fig.9. - Correlation function $P_{0}$ and $P_{1}$ versus the distance $\alpha$ for a $64 \times 64$ square lattice (see text) for: a) $T=0.95$; b) $T=1.01$

these points could be points like the so-called "disorder points" introduced by Stephenson [15]. For a fixed value of $\Delta$ the integrability curve defines a temperature below which the correlation functions could be oscillating with an exponential damping, while above one would have have the usual exponential decay, these oscillating behaviors being not incompatible with our numerical results. 


\section{Acknowledgements.}

When we started our first numerical calculations on the three-state chiral Potts model, we had numerous discussions with R.Rammal. He used to see numerical experiments as "real" experiments, and he liked to find physical interpretations for any new calculations. For this reason our discussions were very stimulating.

\section{References}

[1] Selke.W, "The ANNNI model, Theoretical analysis and experimental application", Phys. Rep. (1988).

[2] H. Au-Yang, B. McCoy, J. Perk, S. Tang and M. Yan, Phys. Lett. A 123, 219 (1987).

[3] McCoy B.M.,Perk J.H.H, Tang. S and Sah C.H., Phys. Lett. A 125, 9 (1987).

[4] Baxter R.J., Perk J.H.H., Au Yang H., Phys. Lett. A 128, 138 (1988).

[5] R. Baxter "Corner transfer matrices of the chiral Potts model. II. The triangular lattice" submitted to J. Stat. Phys. (1992).

[6] J.C. Anglès d'Auriac, D. Hansel, J.M. Maillard, J. Phys A : Math. Gen. 222577 (1989).

[7] Barber M.N 1988 in: Phase Transitions and Critical Phenomena, Vol.8, Eds. C.Domb and J.L. Lebowitz (Academic, New York).

[8] J.C. Anglès d'Auriac, J.M. Maillard, F Wu Physica A 177114 (1991).

[9] Huse D.A, 1981, Phys Rev B24, 5180-5194.

[10] J.C. Anglès d'Auriac, J.M. Maillard, F Wu Physica A 179497 (1991).

[11] Wu. F.Y and Wang Y.K 1976, J. Math. Phys 17, 439.

[12] Mc Coy B.M,.Perk J.H.H, Tang. S and Sah C.H,1987, Phys Lett A125,9

[13] Wu. F.Y,1982, Rev. Mod. Phys. 54, 235

[14] L.Kadanoff Phys. Rev. B22 1405 (1980).

M. Kohmoto, N. den Nijs and L. Kadanoff, Phys, Rev. B24 5829 (1981).

[15] J. Stephenson, J. Math. Phys. 11 413-431 (1970). 